\title{
Uma proposta de análise funcional da aquisição da linguagem: resultados iniciais ${ }^{1}$
}

\author{
Carlos Barbosa Alves de Souza \\ Universidade Federal do Pará
}

\begin{abstract}
Resumo
Dois estudos longitudinais investigaram os efeitos do simples emparelhamento palavra-objeto (i.e., sem o reforçamento de qualquer resposta) na aquisição da competência lingüística de apontar em crianças entre os 7 e 10 meses, e da competência lingüística de nomear em crianças entre os 10 e 13 meses. Verificou-se que o emparelhamento palavra-objeto (a simples exposição ao uso normal de palavras em relação aos objetos) não foi condição suficiente para aprender aquelas competências. No entanto, em ambos estudos se verificou a aquisição gradual das respostas de 'olhar-conjunto' (gazing), imitação motora e vocal, comportamentos que geralmente são sugeridos como pré-requisitos para a aquisição da linguagem. Estes resultados são discutidos considerando a função do emparelhamento palavra-objeto, do reforçamento das respostas e dos comportamentos pré-requisitos na aquisição das competências lingüística. Discute-se uma possível dinâmica de interação entre processos tipo-operante e tipo-respondente na aquisição da linguagem.

Palavras-chave: aquisição da linguagem; análise funcional; emparelhamento palavra-objeto; processos tipo-operante/tipo-respondente.
\end{abstract}

\begin{abstract}
A proposal of functional analysis of the language acquisition: first results

Two longitudinal studies investigated the effects of the word/object pairing (WOP), without reinforcement of any response, in the learning of linguistic competences by infants: 1) during the 7 to 10 month period (competence to point under linguistic criterion); and 2) during the 10 to 13 month period (competence to name). By means of WOP, children did not learn competences. However, in both studies a gradual acquisition of gazing, gesture and vocal imitation responses were verified. These results are discussed in terms of the functionality of WOP, reinforcement of responses, and precurrent behaviors in acquisition of linguistic competences. A possible dynamic of interaction between operant-type and respondent-type processes in language acquisition is discussed.

Keywords: language acquisition; functional analysis; word-object pairing; operant/respondent-type processes.
\end{abstract}

Com o objetivo de estudar a aquisição e o desenvolvimento lingüístico, reconhecendo o caráter multívoco da linguagem, vem se desenvolvendo uma proposta de análise (Souza, 2001; submetido) que parte da noção de 'jogos de linguagem' de Wittgenstein (1953). Essa noção sugere que aprender uma linguagem é aprender diferentes jogos de linguagem, ou seja, é aprender a usar o léxico de um idioma em contextos. "A expressão "jogo de linguagem' procura destacar o fato de que falar uma língua é parte de uma atividade ou de uma 'forma de vida" (Wittgenstein, 1953, pp. 12, 23).

Linguagem como 'uma forma de vida' é uma noção que envolve práticas com objetos e pessoas como uma totalidade integrada, no sentido de que a linguagem está presente e articula qualquer tipo de atividade social imaginável. $O$ ambiente humano, na forma de cultura e de relações sociais, é construído através da linguagem, e nenhuma prática comportamental humana pode ser compreendida apartada da linguagem (Ribes \& Quintana, 2003, p. 442).

Desta maneira, esta proposta parte da concepção de que a aquisição e o desenvolvimento lingüistico ocorrem em função do ensino (informal e/ou formal) que certa comunidade lingüística faz dos usos e contextos de usos de expressões que constituem o léxico desta comunidade, estabelecendo assim diferentes competências lingüísticas, ou seja, respostas convencionalmente determinadas que permitem o desligamento/dis-tanciamento funcional do ambiente imediato (Moerk, 1985; 1996; Ribes, 1990).

A literatura sobre aquisição e desenvolvimento da linguagem sugere que as respostas de imitação, compreensão e produção são as que se aprendem primeiro, e que parecem funcionar como base para o desenvolvimento de outras respostas lingüísticas (Bates, O'Connell, y Shore, 1987; Horne y Lowe, 1996). Entretanto, estas respostas implicam categorias 
demasiadamente amplas, onde diferentes competências lingüísticas podem estar envolvidas em cada tipo de resposta geral. Por isso, se propôs investigar a aquisição específica de três competências que parecem ser parte destas respostas gerais: 1) repetir vocalizações (repetir a morfologia de uma resposta lingüística de maneira exata, reduzida, expandida ou combinada - ver Kuczaj II, 1982); 2) apontar baixo critério lingüísticos (responder de maneira motora e/ou observacional aos produtos lingüísticos de um falante competente); e 3) nomear (produzir uma resposta que designa determinado aspecto do mundo) $)^{2}$.

Inicialmente, procurou-se analisar a competência de repetir sob instruções na presença dos objetos mencionados, e as competências de apontar e nomear em relação a objetos como exemplares, porque estas parecem ser as formas nas quais as respostas gerais, onde podemos identificar estas competências, são ensinadas/aprendidas (Leal, 2000; Macwhinney, 1987; Nelson, 1973) ${ }^{3}$. Concentrou-se em dois aspectos que são mencionados como condições suficientes para a aquisição daquelas respostas lingüísticas: 1) a modelagem e a produção de consequiências às respostas das crianças - reforçamento; e 2) o uso normal de palavras em relação a objetos, sem ensinar nada explicitamente às crianças - emparelhamento palavras-objetos (Horne \& Lowe, 1996).

De maneira congruente com a noção de que aprender uma linguagem é aprender a utilizar certo léxico em contextos, procuramos estabelecer uma análise funcional desta atividade, ou seja, tratamos de estabelecer uma análise que especifique as ações do falante competente, do aprendiz/escuta e os contextos (organização espaço-temporais dos estímulos ambientais) onde estas ocorrem, verificando as relações que se estabelecem entre ações e entre estas e os contextos (Souza, 2001).

Com base em todo isto, estabeleceu-se uma série de estudos (com crianças de 2 anos de idade) que tinham como objetivos: 1) verificar se o emparelhamento palavras-objetos era condição suficiente para a aquisição daquelas três competências lingüísticas; e 2) verificar o efeito da aprendizagem de uma competência sobre o desempenho de outra.

Os resultados destes estudos indicaram que 1) uma situação de uso normal de palavras em relação a objetos (sem ensinar nada às crianças) foi condição suficiente para a aprendizagem das competências de apontar e nomear; 2) as crianças a esta idade já tinham plenamente estabelecida a capacidade de repetição vocal; e 3) o ensino das competências de repetir vocalmente e de apontar não favoreceram a aprendizagem da competência de nomear. Os efeitos do emparelhamento pareceram ser modulados pelo seu número (especialmente no caso de nomear), a sua ordem de apresentação e pelo intervalo entre os emparelhamentos e os "testes de aquisição" (Souza, 2001).

De uma maneira geral, os resultados iniciais desta linha de investigação indicaram, no campo conceptual, a exequiibilidade de se estudar os processos de aquisição e desenvolvimento lingüístico em termos da noção de 'jogos de linguagem' de Wittgenstein (1953). No campo empírico, os resultados sugeriram que o emparelhamento palavrasobjetos, em um contexto de uso funcional de palavras em relação a objetos, parecia ser condição suficiente para a aquisição das competências de apontar e nomear objetos. No entanto, considerando que crianças da idade das que participaram nos estudos (2 a 3 anos) podiam já desempenhar as competências em certo grau, poderia ser que na aprendizagem das competências o emparelhamento tivesse atuado mais no sentido de possibilitar a aprendizagem de respostas específicas; sendo que no caso de nomear, o componente morfológico da resposta poderia ser o elemento principal que os sujeitos tiveram que aprender.

Esta hipótese sugeriu a necessidade de realizar estudos longitudinais com crianças mais novas. Este trabalho descreve dois estudos longitudinais que investigaram os efeitos do emparelhamento palavraobjeto, sem o reforçamento de qualquer resposta, na aquisição de competências lingüísticas de crianças: 1) do $7^{\circ}$ ao $10^{\circ}$ mês (competência de apontar); e 2) do $10^{\circ}$ ao $13^{\circ}$ mês (competência de nomear).

\section{MÉTODO}

Sujeitos: participaram do estudo oito (8) crianças, sem problemas de saúde, de uma pré-escola particular de Lisboa, Portugal. Elas foram designadas aleatoriamente para dois grupos experimentais apontar e nomear (ver Tabela 1 abaixo, onde também se pode ver sexo e idade dos sujeitos).

Equipamento e material: utilizou-se uma câmara de vídeo $(8 \mathrm{~mm})$ operada por controle remoto e dois objetos geométricos de cores diferentes (medindo $10 \mathrm{x}$ $14 \mathrm{~cm} \mathrm{e} 12$ x $14 \mathrm{~cm}$, respectivamente. Ver Anexo 1). Os objetos foram designados com nomes arbitrários (BIPO e TUPA), compostos de duas sílabas de fácil aquisição e pronunciação em português (Martins, 1998).

\begin{tabular}{llc}
\multicolumn{3}{c}{ Tabela 1: Crianças por grupo experimental, idade e sexo. } \\
\hline \multicolumn{1}{c}{ Criança } & \multicolumn{1}{c}{ Idade no início do estudo } & Sexo \\
\hline 1 Apontar & 7 meses e 10 dias & Masculino \\
2AP & 7 meses e 4 dias & Feminino \\
$3 \mathrm{Ap}$ & 7 meses e 2 dias & Feminino \\
$4 \mathrm{AP}$ & 7 meses e $18 \mathrm{dias}$ & Masculino \\
$1 \mathrm{Nomear}$ & 10 meses & Masculino \\
2N & 10 meses e 5 dias & Masculino \\
$3 \mathrm{~N}$ & 10 meses e 12 dias & Feminino \\
4N & 10 meses e 10 dias & Feminino \\
\hline
\end{tabular}




\section{ANEXO 1 \\ Objetos utilizados no estudos sobre as competências de apontar e de nomear}

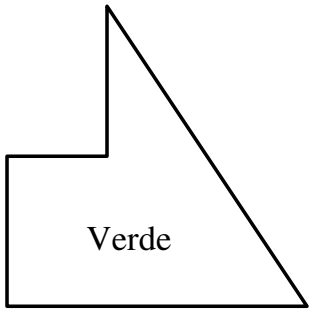

BIPO

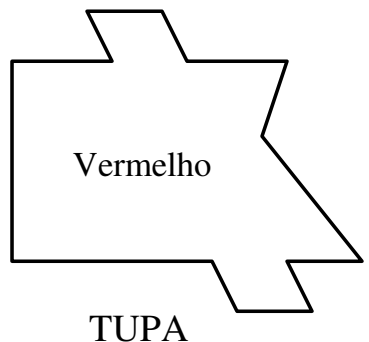

Fase de teste: realizava-se aproximadamente 40 segundos depois de cada fase de emparelhamento. No estudo de nomeação a professora colocava o objeto utilizado na fase de emparelhamento diante da criança e perguntava "Como se chama isto?", "O que é isto?", “Qual é o nome disto?”. A criança tinha 30 segundos para dar uma resposta verbal com a mesma morfologia usada para designar o objeto na fase de emparelhamento.

No estudo de apontar a professora colocava o objeto utilizado na fase de emparelhamento, e outros dois objetos, diante da criança e perguntava "Qual é X?", “Onde está X?". A criança tinha 30 segundos para tomar ou apontar o objeto que tinha sido emparelhado com o nome designado na fase de emparelhamento. Se a criança desse alguma resposta (correta ou não) o teste era repetido, mudando a posição dos objetos.

\section{RESULTADOS E DISCUSSÃO}

Verificou-se que por meio do emparelhamento palavra-objeto as crianças não aprenderam as competências de apontar e nomear. Este resultado concorda com algumas propostas que defendem a necessidade de reforçamento para a aquisição do comportamento verbal (e.g., Skinner, 1957; Moerk, 1999; Horne \& Lowe, 2000).

Por outro lado, em ambos os estudos se observou a aquisição gradual de respostas de observação conjunta (gazing - olhar na mesma direção da professora por mais de 5 segundos), de imitação motora (produzir uma resposta com uma morfologia similar a um comportamento motor da professora, menos de 10 segundos depois deste) e de imitação vocal (produzir uma resposta com uma morfologia similar a um comportamento vocal da professora menos de 10 segundos depois deste). As Figuras 1 a 8 mostram estes resultados (concordância entre observadores de 90 a $100 \%$ para as três respostas). por outro ciclo de emparelhamento-teste com o outro objeto.

Figuras 1 a 8: número de respostas de observação conjunta (gazing), imitação motora e imitação vocal por semana para cada sujeito. Cada ponto representa o total de respostas nas três sessões de uma semana. 


\section{Figura 1}

Sujeito 1AP (Apontar)

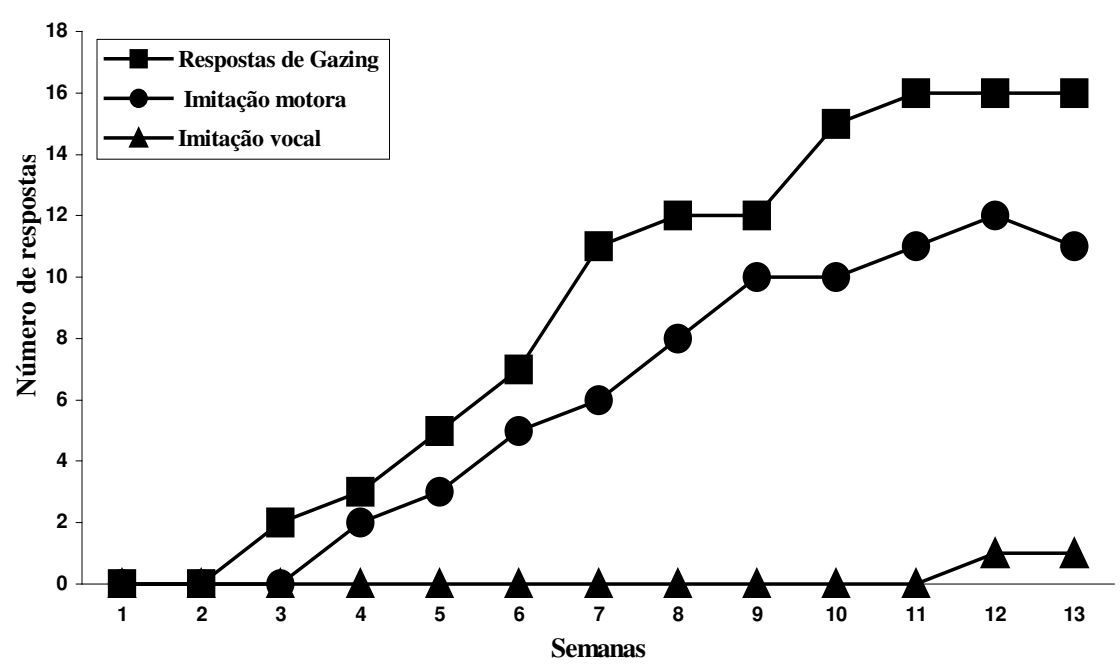

Figura 2

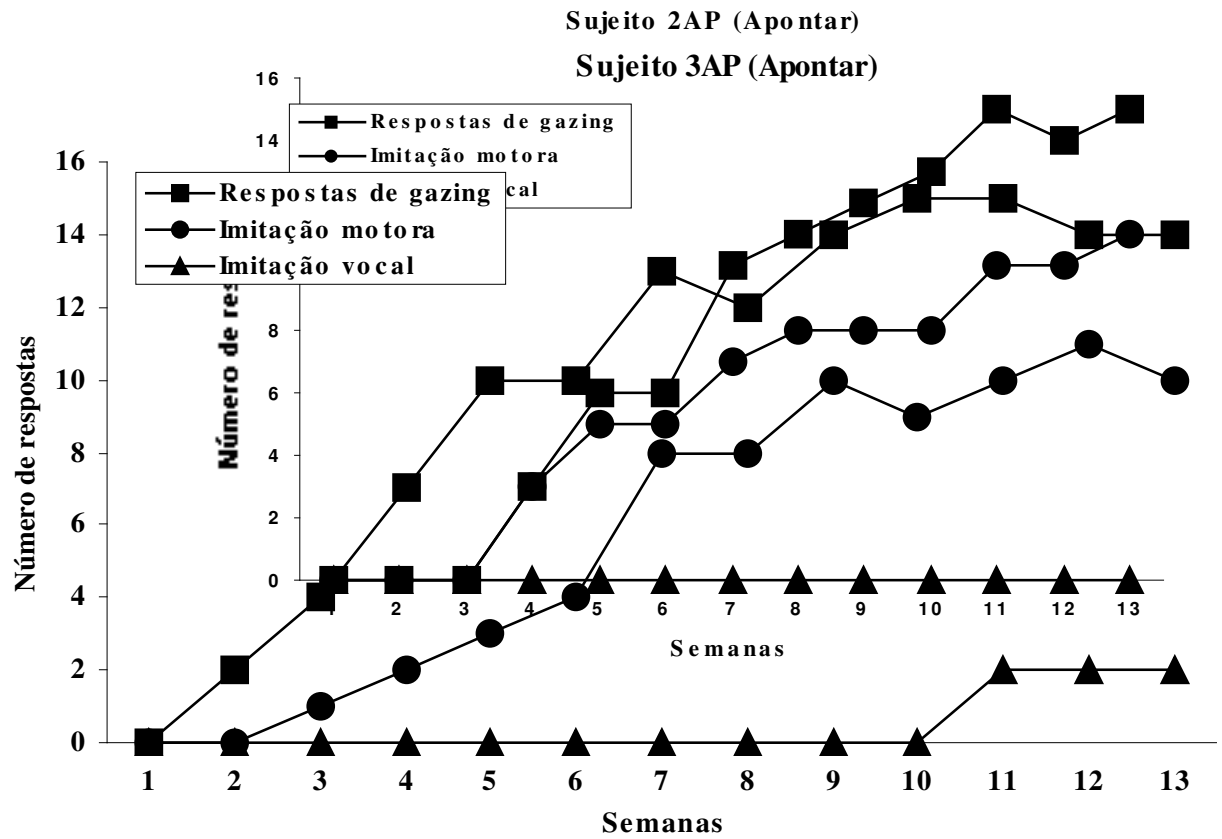

Figura 3

Figura 4 
Sujeito 4AP (Apontar)

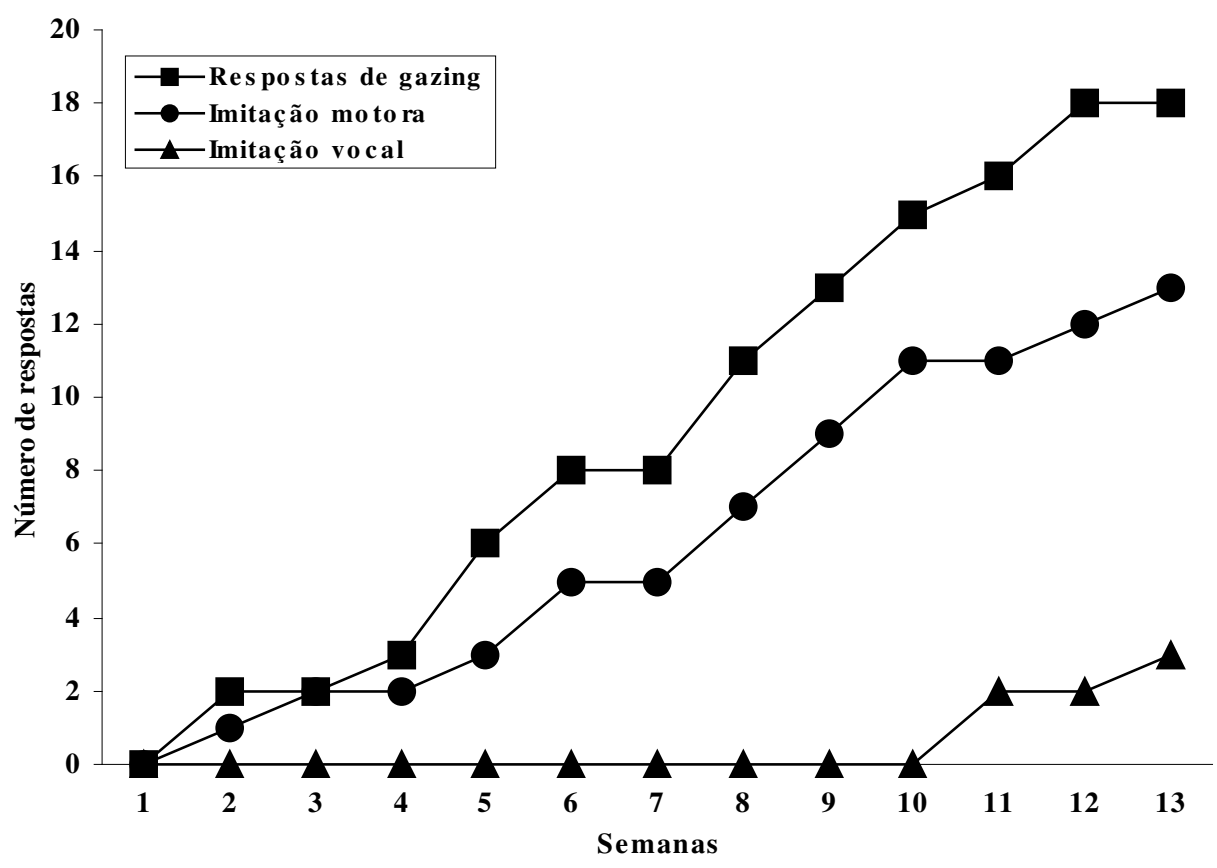

Figura 5

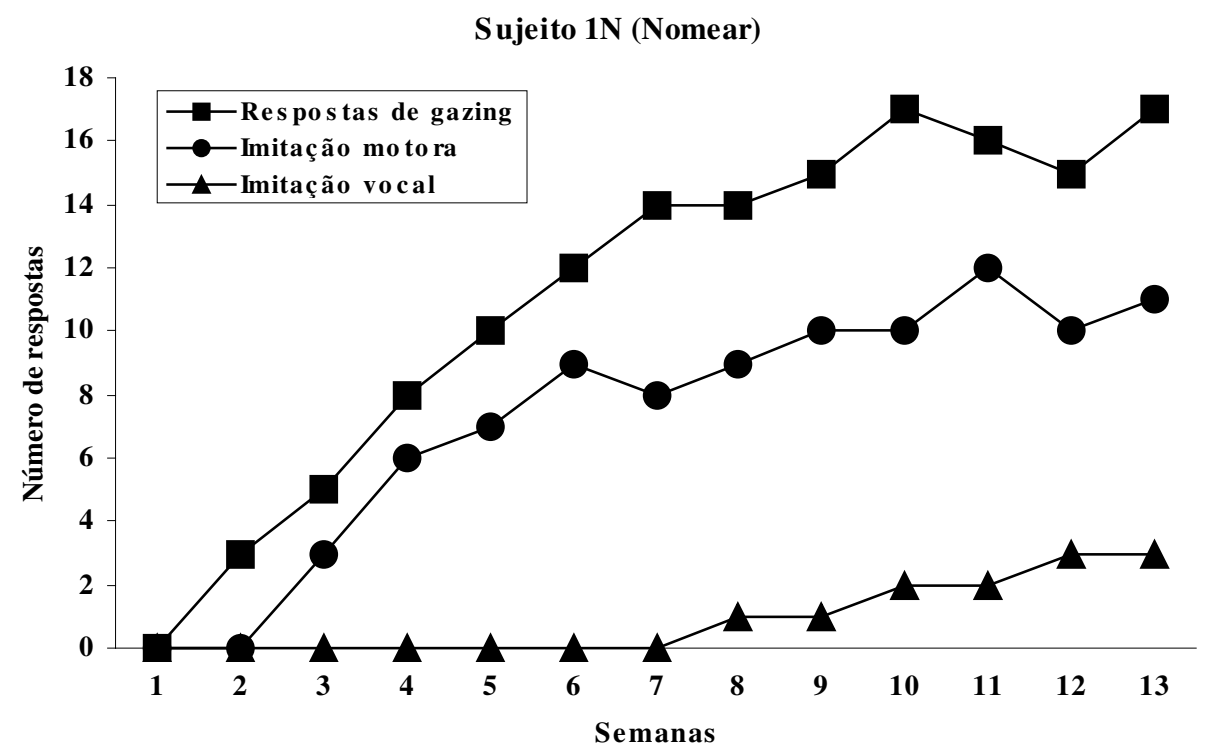

Figura 6 
Sujeito 2N (Nomear)

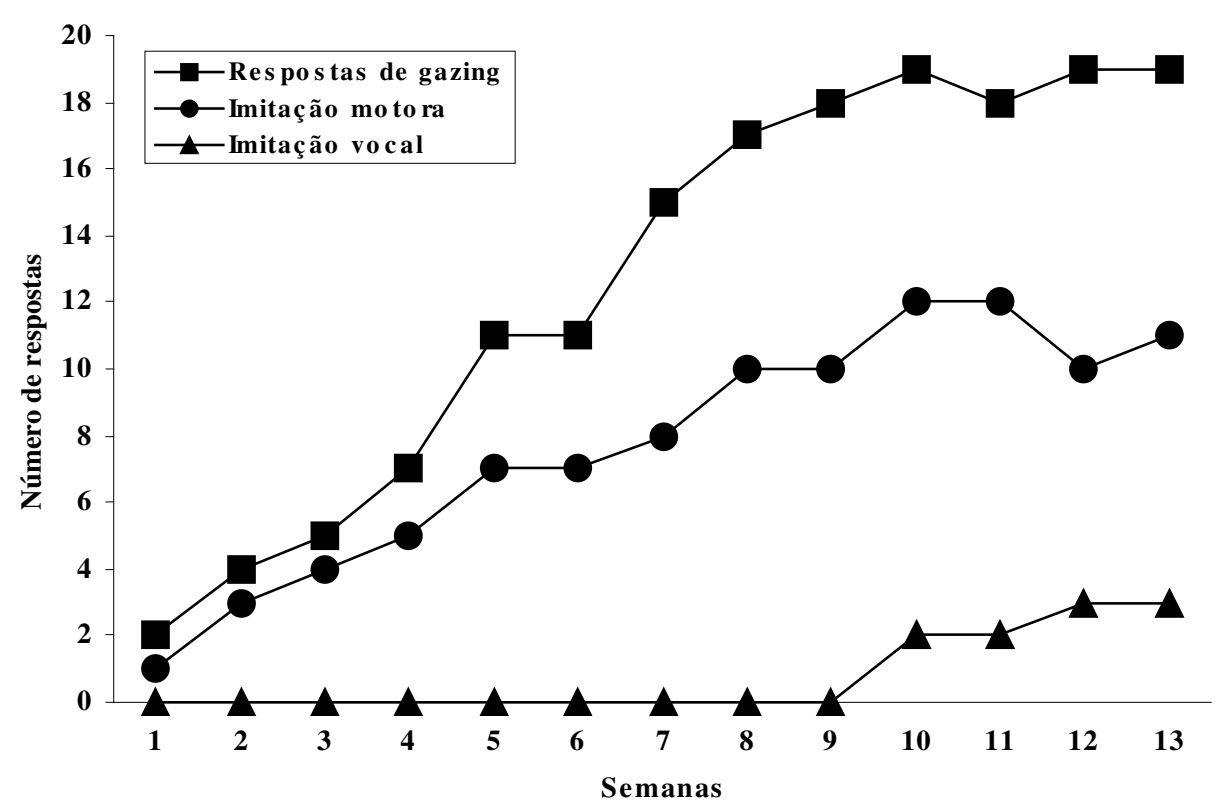

Figura 7

Sujeito 3N (Nomear)

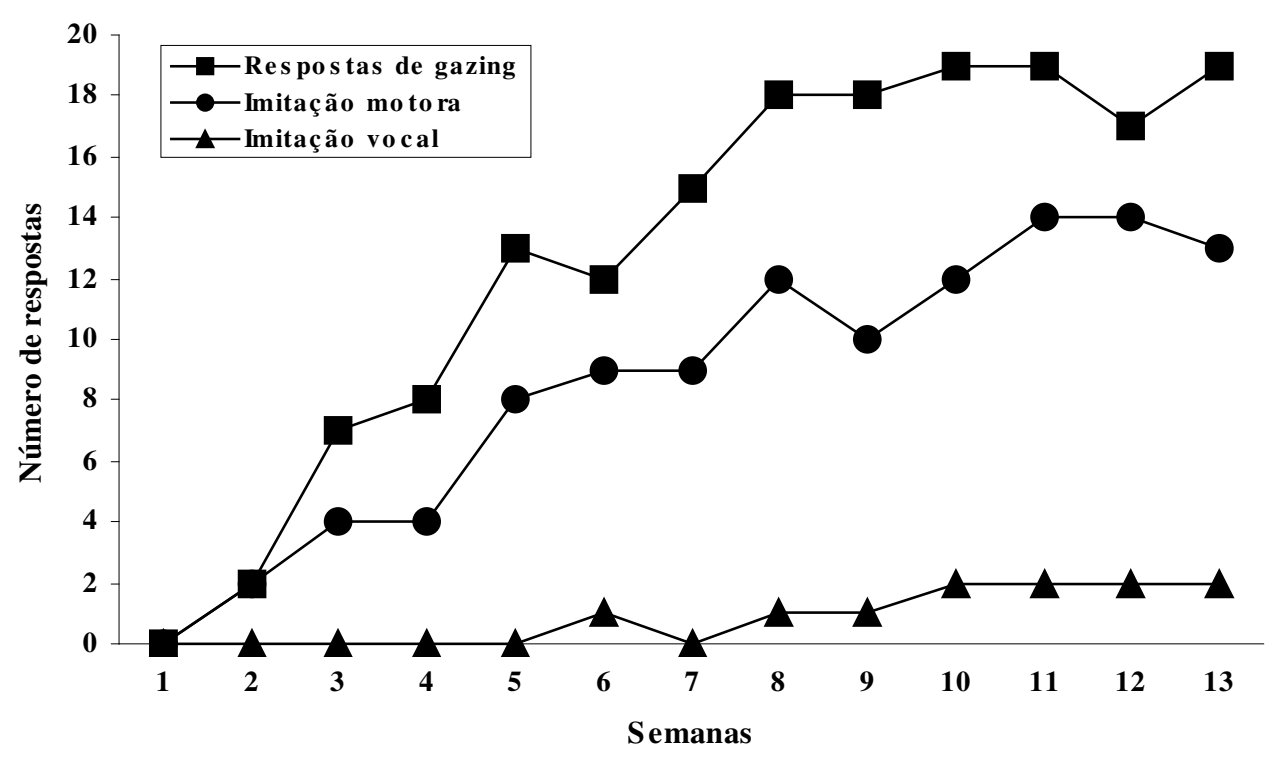




\section{Figura 8}

\section{Sujeito 4N (Nomear)}

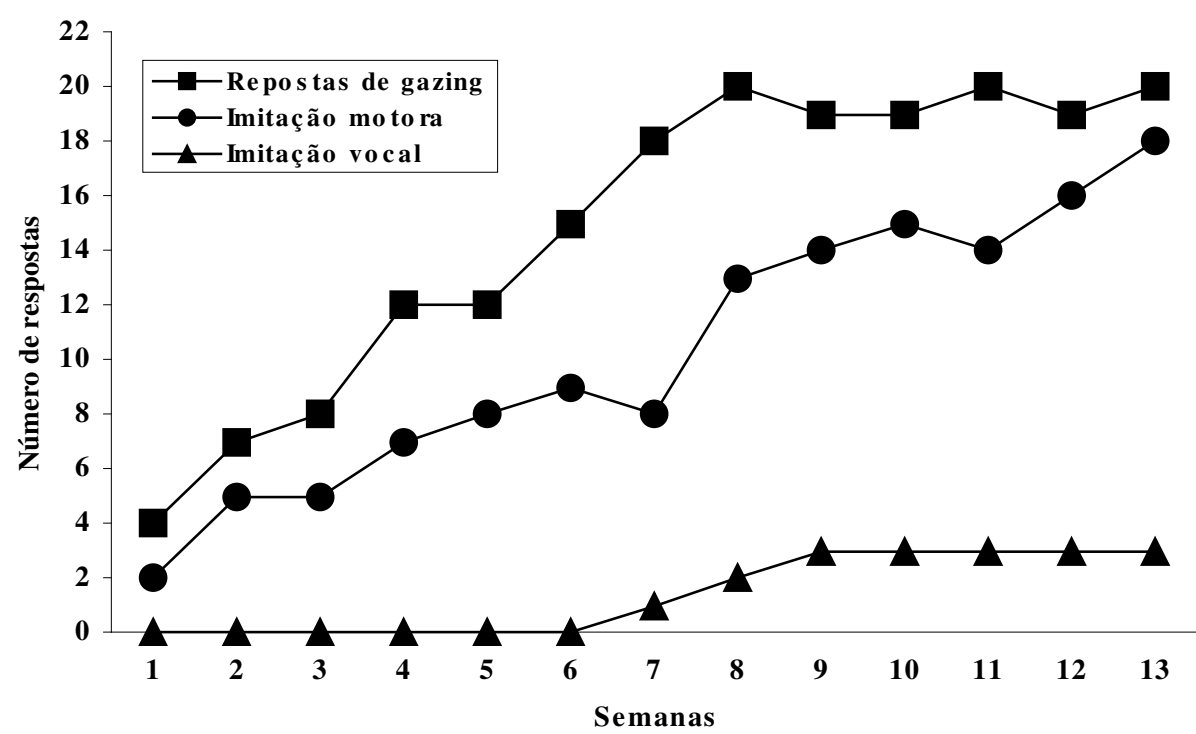

Considerando estes resultados e os resultados positivos obtidos em outros estudos que usaram o emparelhamento palavra-objeto (com ou sem retraso) como procedimento na aquisição de competências lingüísticas por crianças de 18 a 24 meses (Baldwin, 1993; Lucariello, 1987; Moore, Angelopoulos \& Bennett, 1999; Souza, 2001; Tomasello \& Barton, 1994), e de 13 a 18 meses (Shafer e Plunkett, 1998; Woodward, Markman e Fitzsimmons, 1994) parece plausível supor que no período inicial da aquisição de competências lingüísticas (primeiros 24 meses) processos tipo-operante e tipo-respondente atuam de forma sincrônica (ver Alcaráz \& Martínez-Casas, 2000).

Assim, como os resultados deste estudo demonstraram, as situações onde as pessoas usam as palavras em relação com os objetos/eventos do mundo (situações de emparelhamento) podem ser um importante fator na aquisição de comportamentos que são caracterizados como pré-requisitos na aquisição de competências lingüísticas (Butterworth \& Grover, 1988; Luciano \& Polaino-Lorente, 1986; Sonnenschein \& Whitehurst, 1984).

Ao mesmo tempo, as situações de interações onde alguém providencia conseqüências para as respostas promovem a aquisição de classes de respostas (competências) linguiísticas (Moerk, 1999, Stemmer, 1992); que podem ser ampliadas posteriormente (aquisição de morfologias específicas) novamente por meio dos eventos de emparelhamento (ver 'eventos ostensivos' em Stemmer, 1992).

Portanto, a aquisição da linguagem pode ser, no seu estágio inicial, uma atividade sincrônica entre processos tipo-operante (como sugerido por Moerk,
1996) e processos tipo-respondente (como sugerido por Alcaráz e cols., 2000; Stemmer, 1992). Os primeiros resultados analisados de um estudo longitudinal que acompanhou a aquisição da linguagem durante o primeiro ano de vida de um grupo de crianças (Souza, 2002), procurando identificar e analisar os processos e procedimentos implicados nos ajustes funcionais (como sugeridos em Ribes, 2000 e Ribes \& Quintana, 2003) que podem se desenvolver como conseqüências das interações que a criança estabelece com o seu ambiente, parecem favorecer a idéia de uma atividade sincrônica conforme mencionado antes.

\section{REFERÊNCIAS}

Alcaraz, V. M. \& Martínez-Casas, R. (2000). Antes del lenguaje ¿qué?. Em V. M. Alcaraz (Org.), Una mirada múltiple sobre el lenguaje (pp. 161-188). Guadalajara:Universidad de Guadalajara.

Baldwin, D. A. (1993). Early referential understanding: Infants' ability to recognize referential acts for what they are. Developmental Psychology, 29, 832-843.

Bates, E.; O’Connell, B. \& Shore, C. (1987). Language and communication in infancy. Em J. D. Osofsky (Org.), Handbook of Infant Development (pp. 149-203). New York: John Wiley \& Sons.

Butterworth, G. \& Grover, L. (1988). The origins of referential communication in human infancy. Em L. Weiskrantz (Org.), Thought without language (pp. 524). N.Y.: Oxford University Press.

Horne, P. J. \& Lowe, C. F. (2000). Putting the naming account to the test: preview of an experimental program. Em J. C. Leslie \& D. Blackman (Orgs.), Experimental and applied analysis of human behavior (pp. 127-148). Reno, Nevada: Context Press. 
Horne, P. J. \& Lowe, C. F. (1996). On the origins of naming and other symbolic behavior. Journal of the Experimental Analysis of Behavior, 65, 185-241.

Leal, F. (2000). Diez preguntas sobre el lenguaje y un intento por responderlas desde una perspectiva principalmente sintáctica. Em V. M. Alcaraz (Org.), Una mirada múltiple sobre el lenguaje (pp. 33-92). Guadalajara: Universidad de Guadalajara.

Lucariello, J. (1987). Concept formation and its relation to word learning and use in the second year. Journal of Child Language, 14, 309-332.

Luciano, M.C. \& Polaino-Lorente, A. (1986). Effects of the acquisition of prerequisite behavior on the learning of nonvocal verbal behavior and vocal imitation in children with severe retardation. The Psychologica Record, 36, 315-332.

Kuczaj II, S. A. (1982). Language play and language acquisition. Em H. W. Reese (Org.), Advances in Child Development and Behavior: Vol. 17 (pp. 197-232). New York: Academic press.

MacWhinney, B. (1987). Mechanisms of language acquisition. Hillsdale, NJ: Lawnrence Erlbaum.

Martins, M. R. D. (1998). Ouvir falar. Introdução à fonética do português. Lisboa: Caminho.

Moerk, E. L. (1985). The study of language from a skill learning perspective. Em K. Salzinger (Org.), How shall we study verbal behavior. Simpósio apresentado em The Annual meeting of The American Psychological Association, Los Angeles.

Moerk, E. L. (1996). Input and learning processes in first language acquisition. Em H. W. Reese (Org.), Advances in Child Development and Behavior. Vol. 26. (pp. 181228). New York: Academic press.

Moerk, E. L. (1999). Sequential analyses, multiple controlling stimuli, and temporal patterning in firstlanguage transmission. The Analysis of Verbal Behavior, 16, 63-80.

Moore, C.; Angelopoulos, M. \& Bennett, P. (1999). Word learning in the context of referential and salience cues. Developmental Psychology, 35, 60-68.

Nelson, K. E. (1973). Structure and strategy in learning to talk. Monograph of the Society for Research in Child Development, 38, (1\& 2, Serial number 49).

Rescorla, R. A. (1967). Pavlovian conditioning and its proper control procedures. Psychological Review, 74, 71-80.

Ribes, E. (1990). El lenguaje como conducta: Mediación funcional versus descripción morfológica. Em E. Ribes, Psicologia General (pp. 147-176). México: Trillas.
Ribes, E. (2000). El lenguaje desde la perspectiva del estudio del comportamiento: un análisis interconductual. Em V. M. Alcaraz (Org.), Una mirada múltiple sobre el lenguaje (pp. 225-239). Guadalajara: Universidad de Guadalajara.

Ribes, E. \& Quintana, C. (2003). Mother-child linguistic interactions and behavioral development: A multidimensional observational. The Behavior Analyst Today, 3, 442-454.

Schafer, G. \& Plunkett, K. (1998). Rapid word learning by fifteen-month-olds under tightly controlled conditions. Child Development, 69, 309-320.

Sonnenschein, S. \& Whitehurst, G. J. (1984). Developing referential communication: a hierarchy of skills. Child Development, 55, 1.936-1.945.

Souza, C. B. A. (2001). Adquisición de competencias lingüísticas: una propuesta de análisis funcional. Tese de doutorado. Doutorado em Ciências do Comportamento. Centro de Estudios e Investigaciones en Comportamiento, Universidad de Guadalajara. Guadala-jara, Jalisco, México.

Souza, C. B. A. (2002). Aquisição da linguagem: análise conceitual e empírica. Projeto de pesquisa de pósdoutorado (SFRH/BPD/3613/2000 - Fundação para a ciência e a tecnologia de Portugal). Instituto de Filosofia da Linguagem, Universidade Nova de Lisboa. Trabalho não publicado.

Souza, C. B. A. (submetido). Adquisición del lenguaje: enfoques teóricos y una propuesta de análisis. Artigo submetido à Revista Mexicana de Análisis de la Conducta.

Stemmer, N. (1992). The behavior of the listener, generic extensions, and the communicative adequacy of verbal behavior. The Analysis of Verbal Behavior, 10, 69-80.

Stemmer, N. (1996). Listener behavior and ostensive learning. Journal of the Experimental Analysis of Behavior, 65, 247-249.

Tomasello, M. \& Barton, M. (1994). Learning words in nonostensive contexts. Developmental Psychology, 35, 639-650.

Wittgenstein, L. (1953). Philosophical Investigations. New York: Macmillan

Woodward, A. L.; Markman, E. M. \& Fitzsimmons, C. M. (1994). Rapid word learning in 13 and 18 month-olds. Developmental Psychology, 30, 553-566.

Recebido: 09.07.2003

Revisado: 20.07.2003 Aceito:25.07.2003

\footnotetext{
Notas:

${ }^{1}$ Este trabalho foi financiado pela Fundação para a Ciência e a Tecnologia - Ministério da Ciência e do Ensino Superior de Portugal

${ }^{2}$ Ver Souza (2001) para uma descrição mais detalhada da lógica de definição destas competências

${ }^{3}$ Com o desenvolvimento desta linha de análise, investigar-se-ão também a habilidade de repetir sem instruções e as competências de apontar e nomear em relação a i) propriedades de objetos/eventos; ii) classes de objetos/eventos; y iii) relações entre objectos/eventos.
} 


\section{Sobre o autor}

Carlos Barbosa Alves de Souza: Doutor em Ciências do Comportamento (Centro de Estudios e Investigaciones en Comportamiento Universidad de Guadalajara - México). Afiliação: Departamento de Psicologia Experimental, Universidade Federal do Pará. Endereço para correspondência: Universidade Federal do Pará, Centro de Filosofia e Ciências Humanas, Departamento de Psicologia Experimental, Rod. Augusto Correia, s/n, Guamá, 66075-900. Belém, PA. Tel. (91) 211-1662. E-mail: cbas@cpgp.ufpa.br. 

